Journal for ImmunoTherapy of Cancer

\title{
A case of dual-mechanism immune- related anaemia in a patient with metastatic melanoma treated with nivolumab and ipilimumab
}

\author{
Daniel J Olson (1) , ${ }^{1}$ Padma Rajagopal, ${ }^{1}$ Melissa Y Tjota, ${ }^{2}$ Girish Venkataraman, ${ }^{2}$ \\ Jason J Luke, ${ }^{3}$ Thomas F Gajewski ${ }^{1}$
}

To cite: Olson DJ, Rajagopal P, Tjota MY, et al. A case of dualmechanism immune-related anaemia in a patient with metastatic melanoma treated with nivolumab and ipilimumab. Journal for ImmunoTherapy of Cancer 2020;8:e000380. doi:10.1136/jitc-2019-000380

DJO and PR contributed equally.

Accepted 18 December 2019

\section{Check for updates}

(C) Author(s) (or their employer(s)) 2020. Re-use permitted under CC BY-NC. No commercial re-use. See rights and permissions. Published by BMJ.

${ }^{1}$ Medicine, The University of Chicago Medicine Comprehensive Cancer Center, Chicago, Illinois, USA

${ }^{2}$ Pathology, University of Chicago Comprehensive Cancer Center, Chicago, Illinois, USA ${ }^{3}$ Cancer Immunotherapeutics Center, University of Pittsburgh Medical Center Hillman Cancer Center, Pittsburgh, Pennsylvania, USA

Correspondence to Dr Daniel J Olson; dolson2@medicine.bsd. uchicago.edu

\section{ABSTRACT}

Background The combination of the immune checkpoint inhibitors (ICls) ipilimumab and nivolumab is a mainstay of treatment for selected patients with metastatic melanoma. This combination also results in more frequent immunerelated adverse events (irAEs) than either $\mathrm{ICI}$ alone. These irAEs can be severe and their pathogenesis is poorly understood.

Case presentation We report a case of a woman with metastatic melanoma, treated with combined ipilimumab and nivolumab, who developed severe anaemia. While initial workup revealed autoimmune haemolytic anaemia, the anaemia persisted despite corticosteroids and paradoxical reticulocytopenia was observed. Bone marrow biopsy demonstrated a $\mathrm{CD}^{+} \mathrm{T}$ cell-mediated destruction of the red cell precursors implying concurrent pure red cell aplasia. Both processes resolved after the addition of cyclosporine A.

Conclusions This report describes a rare case of two concurrent mechanisms of haematological irAE in a patient treated with combined ICI therapy. Successful treatment resulted only after the second underlying mechanism of toxicity was uncovered. Prompt recognition of these unusual presentations of rare irAEs is now key to effective irAE management.

\section{INTRODUCTION}

The combination of the immune checkpoint inhibitors (ICIs) anti-CTLA-4 (ipilimumab) and anti-PD-1 (nivolumab) has become a mainstay of treatment for selected patients with metastatic melanoma. The use of combination ICIs, while demonstrating higher levels of anti-tumour activity over either ICI alone, also results in more frequent immunerelated adverse events (irAEs). In the Checkmate 067 study, grade 3 and 4 adverse events occurred in $59 \%$ of patients receiving combined ipilimumab and nivolumab, and in $21 \%$ of those receiving nivolumab alone. The most common of these high-grade adverse events were gastrointestinal; however, rare, treatment-related deaths, including myocarditis, hepatic necrosis and neutropaenia were also attributed to ICIs. ${ }^{1}$ Fulminant and life-threatening irAEs, while relatively uncommon, are an ongoing feature of ICI use and can present in unusual fashions. ${ }^{2}$ Prompt recognition of rare irAEs is now key to effective toxicity management.

Haematological irAEs (heme-irAE) represent a rare, but potentially severe subset of irAEs. Whereas irAEs of the skin, gastrointestinal tract, endocrine and musculoskeletal systems are most common, conceivably any organ-including the haematological system-can be affected through an immunemediated toxicity. ${ }^{3}$ Among 948 patients treated with anti-PD-1 and anti-PD-L1 ICIs who were prospectively followed, heme-irAEs occurred in 35 patients. The most common heme-irAEs, occurring in 9/35 patients, were neutropaenia, autoimmune haemolytic anaemia (AIHA), and immune thrombocytopaenia (ITP), while aplastic anaemia occurred in 5/35 patients and pure red cell aplasia (PRCA) was reported in one patient. ${ }^{4}$ In patients treated with anti-CTLA-4 antibodies, severe cases of haemophagocytic lymphohistiocytosis have also been described. ${ }^{5}$ Additionally, a pharmacovigilance study using VigiBase, a WHO database of individual-casesafety-reports found that $12 \%$ of patients had a fatal outcome associated with the identified heme-irAE. ${ }^{6}$ Additionally, 4 of 168 patients in this study experienced overlapping hemeirAEs such as ITP and AIHA, while $23 \%$ of patients with a heme-irAE had a concomitant non-heme irAE.

Cases of overlapping irAEs, though far less common than single events, have been observed across a broad spectrum of irAEs. Among 122 reported cases of ICI-related myocarditis, concomitant myasthenia gravis (MG) and myositis were observed in $11 \%$ and $25 \%$ of cases, respectively. ${ }^{2}$ Similarly, a 
pharmacovigilance study of 228 cases of ICI-related MG reported respective overlapping cases of myocarditis and myositis in $10.5 \%$ and $16.2 \%$ of patients. ${ }^{7}$ This unusual autoimmune presentation had been characterised even prior to the introduction of ICIs. A 2009 study of patients with MG who also developed an autoimmune inflammatory myopathy suggested that there may be an overlapping autoimmune target. Notably, both humoral and cellular mechanisms were implicated: nearly all patients had positive antiacetylcholine receptor antibodies while muscle biopsies demonstrated a monocytic and lymphocytic inflammatory infiltrates. ${ }^{8}$ Similarly, within reports of ICI-related MG and myositis, both humoral and cellular processes are described. ${ }^{29}$ Taken together, these cases of overlapping irAEs demonstrate that multiple concurrent mechanisms of toxicity may exist within a single patient experiencing an irAE.

Here, we present the clinicopathological features of a case of dual-mechanism heme-irAE in a patient with metastatic melanoma treated with combined nivolumab and ipilimumab.

\section{CASE PRESENTATION}

A 29-year-old woman presented to our clinic with progressive fatigue following treatment with ipilimumab and nivolumab for metastatic melanoma. Three years prior, she had undergone superficial excision of a posterior-auricular atypical melanocytic neoplasm, and was subsequently followed through observation alone. One month prior to her initial referral to our clinic, she developed diffuse pruritus with liver function tests demonstrating an ALT of 1166 U/L (range: 0-32 U/L), AST of $281 \mathrm{U} / \mathrm{L}$ (range: 0-32 U/L), alkaline phosphatase of $281 \mathrm{U} / \mathrm{L}$ (range: $35-104 \mathrm{U} / \mathrm{L}$ ) and otherwise normal routine laboratory parameters. She underwent upper endoscopy that showed a $2.2 \mathrm{~cm}$, hyperpigmented mass causing a biliary obstruction at the major papilla. Biliary stenting and a biopsy of the mass were completed. The results were consistent with malignant melanoma; a BRAF V600E mutation was also detected. Baseline crosssectional imaging was completed for staging and no additional sites of disease were identified beyond the mass detected by endoscopy. After referral to our clinic, she was started on combination nivolumab $(1 \mathrm{mg} / \mathrm{kg})$ and ipilimumab $(3 \mathrm{mg} / \mathrm{kg})$ every 3 weeks for four cycles. She developed immune-related primary hypothyroidism after cycle two, for which she began levothyroxine.

She then reported having gradually worsening fatigue and dyspnoea on exertion 3 weeks after completing her fourth dose of nivolumab and ipilimumab. On examination, she was pale appearing and exhibited a regular tachycardic rhythm. She had normal pulmonary and abdominal exams. The heart rate was $117 \mathrm{bpm}$ and blood pressure was 115/79 mm Hg. Haematological parameters revealed a haemoglobin of $61 \mathrm{~g} / \mathrm{Lg} / \mathrm{L}$, decreased from $124 \mathrm{~g} / \mathrm{L} 3$ weeks prior (figure 1); the white cell count

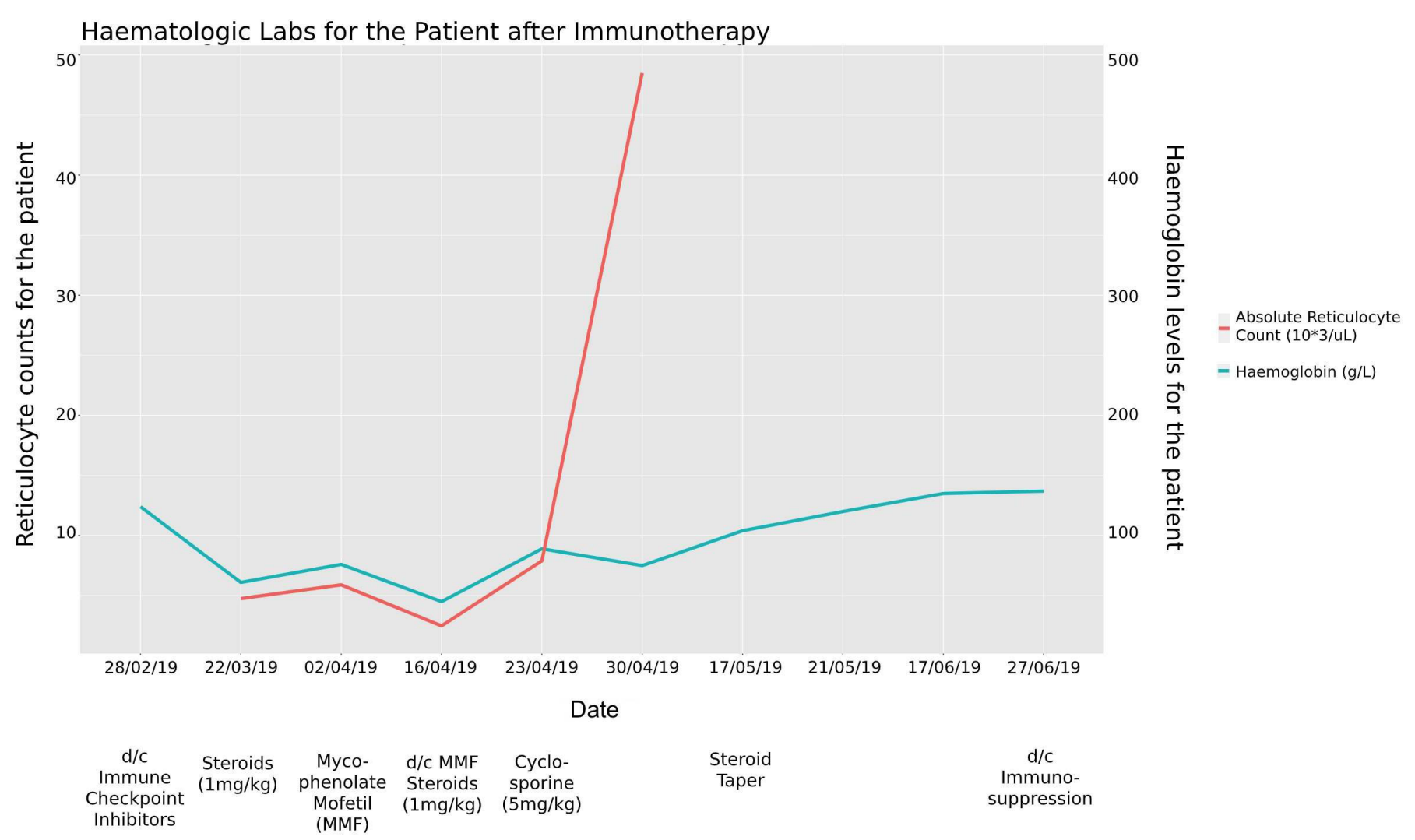

Figure 1 Laboratory trends over time. Haemoglobin and absolute reticulocyte counts are noted. Periods of immunosuppressant administration are listed along the X-axis. 


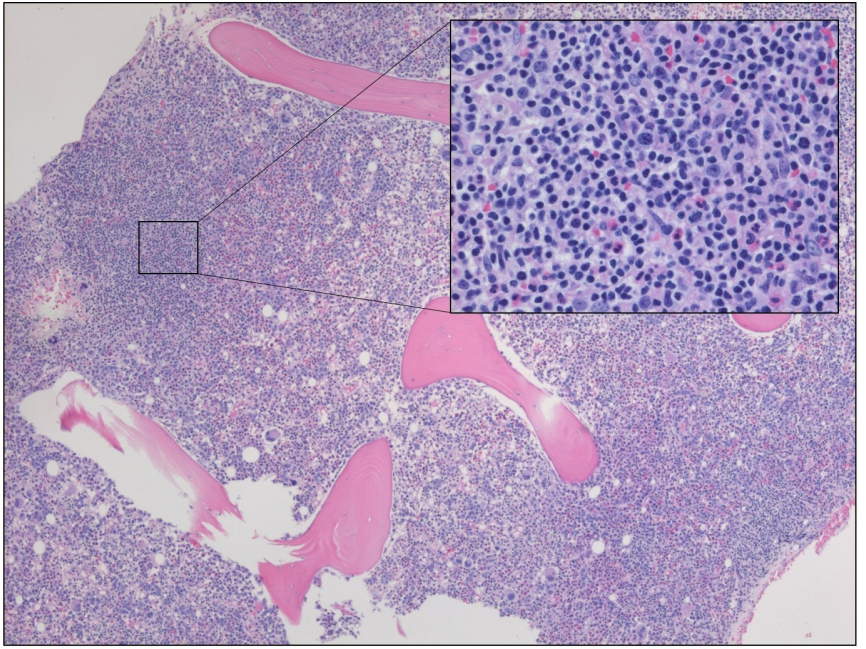

Figure 2 Hypercellular bone marrow core biopsy sections with atypical lymphoid aggregates; H\&E, magnification of $\times 100$ with $\times 500$ insert.

and platelet counts were normal. The mean corpuscular volume was 85 (range 81-99 fL) and the ferritin was elevated to $457 \mathrm{ng} / \mathrm{mL}$ (range $10-220 \mathrm{ng} / \mathrm{mL}$ ). Haemolysis labs demonstrated an undetectable haptoglobin, lactate dehydrogenase of $492 \mathrm{U} / \mathrm{L}$ (range 116-245 U/L) and a strongly positive direct antiglobulin test (Coombs) with elution of broadly specific warm autoantibodies. A presumptive diagnosis of autoimmune haemolytic anaemia (AIHA) was made, and the patient was admitted to the hospital for transfusion support and intravenous corticosteroids. She received two units of packed red blood cells (PRBCs) and $1 \mathrm{~g}$ of methylprednisolone every 12 hours over 2 days. Once symptoms improved and her haemoglobin had stabilised at $79 \mathrm{~g} / \mathrm{L}$, she was discharged home on $1 \mathrm{mg} / \mathrm{kg}$ oral prednisone without a scheduled taper.

One week following discharge, she reported worsening fatigue and dyspnoea on exertion. The haemoglobin was found to be $76 \mathrm{~g} / \mathrm{d}$ with continued evidence of haemolysis,

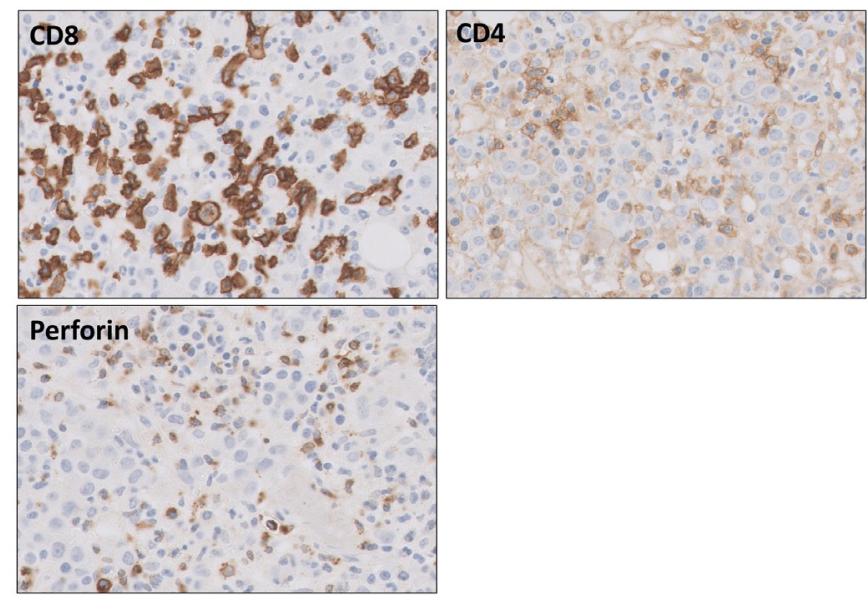

Figure 3 Bone marrow core biopsy. Immunohistochemistry for CD8 (C8/144B, Dako), CD4 (SP35, thermo Fisher) and perforin (5B10, Novocastra), magnification of $\times 400$. and paradoxically, the reticulocyte count was found to be inappropriately low for the degree of anaemia-the absolute reticulocyte count was $5.9 \times 10^{3}$ with a Reticulocyte Production Index (RPI) of 0.0. Steroids were discontinued given their apparent poor control of anaemia. She started mycophenolate mofetil $1000 \mathrm{mg}$ twice per day; the following week her haemoglobin was found to be $64 \mathrm{~g} / \mathrm{L}$ and she was again transfused two units of PRBC, and mycophenolate mofetil was increased to $1500 \mathrm{mg}$ twice per days. Subsequent laboratory testing completed a week later again showed severe anaemia with a haemoglobin of $45 \mathrm{~g} / \mathrm{L}$ and ongoing reticulocytopenia. At this point, she was readmitted to the hospital, intravenous corticosteroids were reinitiated, and a bone marrow biopsy and aspirate were performed. The marrow core biopsy sections were hypercellular with an atypical lymphoid infiltrate and a paucity of early red cell precursors present (figure 2). Twenty-five per cent of all cells stained as $\mathrm{CD} 8^{+}$, and the CD4:CD8 ratio was abnormally shifted to $1: 3$, showing predominance of $\mathrm{CD}^{+} \mathrm{T}$ cells (figure 3). Perforin was also increased suggesting a cytotoxic $\mathrm{T}$ cell phenotype. $\mathrm{T}$ cell receptor gene rearrangement was assessed on the bone marrow aspirate by PCR and showed a polyclonal $T$ cell population. The OncoPlus universal cancer mutation panel at University of Chicago, a next-generation sequencing assay of 147 genes designed for both solid and haematological malignancies, was performed on the bone marrow aspirate. ${ }^{10}$ This did not identify any pathogenic mutations.

These observations were consistent with $\mathrm{CD} 8^{+} \mathrm{T}$ cellmediated PRCA. Given the lack of reticulocyte response to corticosteroids and the short trial of mycophenolate mofetil, T cell-directed immunosuppression was initiated with cyclosporine A ( $5 \mathrm{mg} / \mathrm{kg}$ /day in divided doses) along with continued $1 \mathrm{mg} / \mathrm{kg}$ prednisone administration for AIHA. Within 1 week, the absolute reticulocyte count had increased, and by 3 weeks the absolute reticulocyte count had increased from $2.4 \times 10^{3}$ to $226 \times 10^{3}$ with an RPI of 2.1, reflecting an appropriate bone marrow response to the degree of anaemia. Within 1 month, her haemoglobin had normalised at $120 \mathrm{~g} / \mathrm{L}$ (figure 1 ). The direct antiglobulin test (Coombs) turned negative thereafter. A steroid taper was completed, and all immunosuppression was ceased within 3 months. No further immunotherapy was given after the initial anaemia diagnosis or after resolution of the heme-irAEs. On repeat imaging, the patient experienced a complete response to ipilim$\mathrm{umab} /$ nivolumab and remains in observation alone with continued response.

\section{DISCUSSION}

Currently, corticosteroids are the guideline-directed firstline treatment for nearly all high-grade (ie, grades 3-4) irAEs. ${ }^{11}$ With perhaps the exception of tumour necrosis factor inhibitors, such as infliximab, for ICI-related colitis, additional immunosuppressive regimens have not been evaluated in large numbers of patients. ${ }^{12}$ As a result, 
guidelines for the management of steroid-refractory irAEs frequently recommend referral to subspecialist disciplines for the affected organ, or the phenotypically similar autoimmune/inflammatory disorder. ${ }^{311}$ In such cases-as with rituximab for ICI-related haemolytic anaemia, or methotrexate for ICI-related inflammatory arthritis-the additional immunosuppressive regimens frequently are based on the non-ICI related but clinicallyanalogous disorder.

Our patient had clear evidence of AIHA as demonstrated by the presence of haemolysis and a positive Coombs test. Corticosteroids are the preferred first-line treatment for AIHA, with secondary options for refractory disease including splenectomy or rituximab. ${ }^{13}$ In this case, the persistent anaemia after corticosteroid initiation was apparently due to concurrent failure in marrow production of red cell precursors. Accordingly, empiric addition of a B lymphocyte-directed therapy like rituximab may not have improved red cell production. Instead, the paradoxical reticulocytopenia in the context of AIHA raised concern for a separate process; this was ultimately identified via bone marrow biopsy as $\mathrm{CD}^{+} \mathrm{T}$ cell-mediated red cell aplasia. Therefore, this patient demonstrated two current mechanisms of immune-mediated anaemia: one antibody mediated and the other $\mathrm{CD} 8^{+} \mathrm{T}$ cell mediated.

Acquired PRCA encompasses a heterogeneous group of disorders, all leading to the underproduction of erythrocytes. This includes antibody-mediated destruction of erythroid precursors, as has been observed in primary acquired PRCA, and secondary PRCA, which is frequently associated with autoimmune/collagen vascular disorders and lymphoproliferative disorders-the mechanisms of which may be immunologic, but not necessarily antibody mediated. ${ }^{14}$ One of the most commonly associated disorders with secondary PRCA, large granular lymphocyte (LGL) leukaemia, frequently presents with an interstitial lymphoid infiltrate of the bone marrow. ${ }^{15}$ On initial visualisation of our patient's marrow biopsy, we considered LGL within our differential diagnosis given the morphological appearance of the lymphocytic infiltrate and known association of LGL with autoimmune processes. However, LGL would normally demonstrate clonally rearranged $\mathrm{T}$ cell receptor genes, which was not identified in this case. These results suggested a reactive rather than clonal lymphocytic process. Ultimately, the identification of this $\mathrm{CD}^{+}$-predominant $\mathrm{T}$ cell infiltrate prompted our initiation of cyclosporine A for its preferential inhibition of T lymphocytes. ${ }^{16}$ Notably, cyclosporine A appears to be one of the most effective immunosuppressive regimens for PRCA. From pooled case series across multiple PRCA subtypes, cyclosporine A use, with or without corticosteroids, demonstrated a response rate of over $75 \%$; this is numerically higher than other observed immunosuppressive regimens such as tacrolimus, mycophenolate mofetil and antithymocyte globulin. ${ }^{14} 17$

Despite similarities between irAEs to analogous autoimmune disorders, the underlying pathogeneses of ICI toxicity remains unclear. The CTLA-4 and PD-1 pathways, operating at different stages of an immune response, are central to the maintenance of peripheral tolerance and to the regulation of autoimmunity. As the cancer context leads to dominant activity of these negative immune regulators in many patients, the release of these negative regulators can promote anti-tumour immune recognition and killing. ${ }^{18}$ Immune checkpoint inhibition may also lead to an imbalance of peripheral tolerance in healthy tissues, leading to the collateral inflammation observed across the spectrum of irAEs. Multiple mechanisms have been proposed, including increased $\mathrm{T}$ cell activity against selfantigens, induced antibody-mediated reactivity against tissue proteins, generalised cytokine release and enhanced complement-mediated inflammation. ${ }^{19}$ However, the precise mechanisms by which immune checkpoint inhibition leads to specific irAEs are not yet well understood. Accordingly, our ability to predict which patients are more likely to experience an irAE remains limited. While the presence of an underlying auto-immune disorder confers a higher risk of an irAE, this does not reliably predict the onset or severity of an irAE. ${ }^{20}$ Notably, the rates of high-grade irAEs are broadly similar in the neoadjuvant, adjuvant and metastatic settings for anti-PD-1 and antiCTLA-4 agents, both alone and in combination. ${ }^{21-23}$ This suggests that the determinants of most irAEs are likely host factors, and are less likely related to cancer disease status. Associations between germline variants associated with autoimmune risk, preexisting serum antibodies and composition of the commensal microbiota with the development of irAEs have all been reported, suggesting that they may be patient-intrinsic factors that could guide future biomarkers of toxicity. ${ }^{24-26}$ Further validation in larger cohorts of patients will be required in order to provide clinical utility.

\section{CONCLUSIONS}

This report describes a rare case of two concurrent mechanisms of heme-irAE in a patient treated with combined ICI therapy: (1) autoantibody mediated AIHA and (2) CD8 + T cell-mediated PRCA. Institution of treatment with both corticosteroids and cyclosporine A reversed each of these pathologies and enabled stable recovery of circulating red blood cells. This case highlights both the importance of accurate recognition and diagnosis of rare irAEs as well as the challenges in the management of high-grade, steroid-refractory irAEs. It also reveals our limited understanding of irAE risk factors, while underscoring the research imperative to better characterise the pathogeneses of irAEs to improve our clinical predictive abilities for patients.

\section{Twitter Daniel J Olson @DanielOIsonMD}

Contributors D0 and PR wrote and edited the manuscript and contributed equally to this work. MT and GV performed the histological examination of the bone marrow and provided the figures. DO, PR, JL and TFG coordinated the patient's care. TFG edited and had final editorial control over the manuscript. All authors read and approved the final manuscript. 
Funding This work was supported in part by T32GM007019 (DJO).

Competing interests TFG has received research funding from Bristol-Myers Squibb, the manufacturer of ipilimumab and nivolumab.

Patient consent for publication Not required.

Provenance and peer review Not commissioned; externally peer reviewed.

Open access This is an open access article distributed in accordance with the Creative Commons Attribution Non Commercial (CC BY-NC 4.0) license, which permits others to distribute, remix, adapt, build upon this work non-commercially, and license their derivative works on different terms, provided the original work is properly cited, appropriate credit is given, any changes made indicated, and the use is non-commercial. See http://creativecommons.org/licenses/by-nc/4.0/.

\section{ORCID iD}

Daniel J Olson http://orcid.org/0000-0001-8902-7661

\section{REFERENCES}

1 Wolchok JD, Chiarion-Sileni V, Gonzalez R, et al. Overall survival with combined nivolumab and ipilimumab in advanced melanoma. $N$ Engl J Med 2017;377:1345-56.

2 Salem J-E, Manouchehri A, Moey M, et al. Cardiovascular toxicities associated with immune checkpoint inhibitors: an observational, retrospective, pharmacovigilance study. Lancet Oncol 2018;19:1579-89.

3 Brahmer JR, Lacchetti C, Schneider BJ, et al. Management of immune-related adverse events in patients treated with immune checkpoint inhibitor therapy: American Society of clinical oncology clinical practice guideline. JCO 2018;36:1714-68.

4 Delanoy N, Michot J-M, Comont T, et al. Haematological immunerelated adverse events induced by anti-PD-1 or anti-PD-L1 immunotherapy: a descriptive observational study. Lancet Haematol 2019;6:e48-57.

5 Hantel A, Gabster B, Cheng JX, et al. Severe hemophagocytic lymphohistiocytosis in a melanoma patient treated with ipilimumab + nivolumab. J Immunother Cancer 2018;6:73-7.

6 Davis EJ, Salem Joe-Elie, Young A, et al. Hematologic complications of immune checkpoint inhibitors. Oncologist 2019;24:584-8.

7 Johnson DB, Manouchehri A, Haugh AM, et al. Neurologic toxicity associated with immune checkpoint inhibitors: a pharmacovigilance study. J Immunother Cancer 2019;7:134.

8 Suzuki Set al. Autoimmune targets of heart and skeletal muscles in myasthenia gravis. Arch Neurol 2009;66:1334-8.

9 Gonzalez NL, Puwanant A, Lu A, et al. Myasthenia triggered by immune checkpoint inhibitors: new case and literature review. Neuromuscular Disorders 2017;27:266-8.
10 Kadri S, Long BC, Mujacic I, et al. Clinical validation of a nextgeneration sequencing genomic oncology panel via Cross-Platform benchmarking against established amplicon sequencing assays. J Mol Diagn 2017;19:43-56.

11 Puzanov I, Diab A, Abdallah K, et al. Managing toxicities associated with immune checkpoint inhibitors: consensus recommendations from the Society for immunotherapy of cancer (SITC) toxicity management Working Group. in: vol 5. BioMed Central 2017:95-28.

12 Johnson DH, Zobniw CM, Trinh VA, et al. Infliximab associated with faster symptom resolution compared with corticosteroids alone for the management of immune-related enterocolitis. $J$ Immunother Cancer 2018;6:103-8.

13 Brodsky RA. Warm autoimmune hemolytic anemia. Solomon CG, ED. N Engl J Med 2019:647-54.

14 Means RT. Pure red cell aplasia. Blood 2016;128:2504-9.

15 Agnarsson BA, Loughran TP, Starkebaum G, et al. The pathology of large granular lymphocyte leukemia. Hum Pathol 1989;20:643-51.

16 Matsuda S, Koyasu S. Mechanisms of action of cyclosporine. Immunopharmacology 2000;47:119-25.

17 Wu X, Wang S, Shen W, et al. Adult patients with acquired Pure Red Cell Aplasia: treated by Cyclosporine a and/or Corticosteroids-Single Center Experience. Blood 2016;128:4818.

18 Buchbinder El, Desai A. Ctla-4 and PD-1 pathways: similarities, differences, and implications of their inhibition. Am J Clin Oncol 2016;39:98-106.

19 Pauken KE, Dougan M, Rose NR, et al. Adverse events following cancer immunotherapy: obstacles and opportunities. Trends Immunol 2019;40:511-23.

20 Leonardi GC, Gainor JF, Altan M, et al. Safety of programmed Death-1 pathway inhibitors among patients with Non-SmallCell lung cancer and preexisting autoimmune disorders. JCO 2018;36:1905-12.

21 Robert C, Schachter J, Long GV, et al. Pembrolizumab versus ipilimumab in advanced melanoma. N Engl J Med 2015;372:2521-32.

22 Garcia CA, El-Ali A, Rath TJ, et al. Neurologic immune-related adverse events associated with adjuvant ipilimumab: report of two cases. J Immunother Cancer 2018;6:459.

23 Blank CU, Rozeman EA, Fanchi LF, et al. Neoadjuvant versus adjuvant ipilimumab plus nivolumab in macroscopic stage III melanoma. Nat Med 2018;375:1.

24 Kirchhoff T, Ferguson R, Simpson D, et al. 1147PDGermline determinants of immune related adverse events (irAEs) in melanoma immunotherapy response. Annals of Oncology 2017;28.

25 Toi Y, Sugawara S, Sugisaka J, et al. Profiling preexisting antibodies in patients treated with Anti-PD-1 therapy for advanced Non-Small cell lung cancer. JAMA Oncol 2019;5:376-83.

26 Postow MA, Sidlow R, Hellmann MD. Immune-Related adverse events associated with immune checkpoint blockade. N Engl J Med 2018;378:158-68. 